\title{
Der Einfluss des deutschen Verfassungsrechtsdenkens in der Welt...
}

Selektive Anmerkungen zur Tagung der Gesellschaft für Rechtsvergleichung vom 12.- 13. September in Marburg

Sebastian v. Kielmansegg"

Das Schöne an der Rechtsvergleichung ist, dass man sich mit ihr auf ein Feld intradisziplinärer Interdisziplinarität begibt. Sie ist eine genuin rechtswissenschaftliche Disziplin, aber ihr Blick ist auf das Andere, auf die Erschließung fremder Rechtskulturen gerichtet. So sind auch die Tagungen, die die Gesellschaft für Rechtsvergleichung in zweijährigem Turnus abhält, ein Erlebnis der Vielseitigkeit und Offenheit: länderübergreifend dank der ausländischen Referenten und Mitglieder; fachübergreifend, weil die Rechtsvergleichung als Querschnittsdisziplin die sonst so sorgsam gepflegte Sektorengrenze zwischen Zivilrecht, Strafrecht und Öffentlichem Recht überschreitet; und generationsübergreifend, weil die Gesellschaft für Rechtsvergleichung nicht die Weihe der Habilitation verlangt und deshalb auch den jungen Kollegen offensteht. So ganz gilt das freilich nur für Kaffeepausen und Rahmenprogramm, denn im wissenschaftlichen Teil bleiben die einzelnen Fachgruppen dann doch mehr oder minder unter sich.

Die diesjährige Tagung in Marburg stand unter dem Oberthema „Recht und Rechtsvergleichung in der Finanzkrise“. Diesmal hatte man es damit freilich nicht sehr genau genommen. Nur die Fachgruppen Handels- und Wirtschaftsrecht sowie Europarecht haben sich in ihrer gemeinsamen Sitzung „Finanzmarktregulierung in der Krise" recht eigentlich dieser Thematik angenommen, im weiteren Sinne auch der Festvortrag am Eröffnungsabend, den Thomas von Danwitz zur „Wirtschaftsregulierung in der Rechtsprechung des EuGH“ hielt. Die übrigen Fachgruppen hatten ihre Themen breit gestreut: „Recht und Sozialtheorie - interdisziplinäres Denken in Rechtswissenschaft und -praxis“ (Grundlagen); „Der Schutz dinglicher Rechtspositionen und die Gewährleistung effizienten Rechtsverkehrs“ (Zivilrechtsvergleichung); „Der Einfluss des deutschen Verfassungsrechtsdenkens in der Welt: Bedeutung, Grenzen, Zukunftsperspektiven“ (Vergleichendes Öffentliches Recht); „Die Strafbarkeit juristischer Personen - Selbstverständlichkeit oder Paradigmenwechsel im Strafrecht?“ (Strafrechtsvergleichung); und „Arbeitnehmerüberlassung als Instrument zur Flexibilisierung von Arbeitsmärkten“ (Vergleichendes Arbeits- und Sozialrecht).

Auskosten kann der Teilnehmer diese Bandbreite nur begrenzt, weil die Fachgruppensitzungen parallel abgehalten werden - auch wenn zur Entzerrung nun erstmals eine der Sitzungen auf den Eröffnungstag vorverlegt worden war. Tagungseindrücke

* Prof. Dr. Sebastian Graf Kielmansegg, HEUSSEN-Stiftungslehrstuhl für Öffentliches Recht, Europarecht, Recht der erneuerbaren Energien und Medizinrecht, EBS-Universität Wiesbaden. 
sind daher notwendigermaßen selektiv. Das gilt auch für diese Zeilen, weil dem Verfasser als Mitorganisator einer der Fachgruppensitzungen das eigentlich durchaus erwünschte Sitzungs-Hopping versagt war. So ist es nur das Vergleichende Öffentliche Recht, um dessen Sitzung die weiteren Gedanken kreisen können.

Das Thema stach denn auch etwas hervor: „Der Einfluss des deutschen Verfassungsrechtsdenkens in der Welt“. Was für eine Frage!, könnte man anmerken und ist auch zuweilen angemerkt worden. In der Tat kann den Beobachter leicht ein gewisses Unbehagen beschleichen. Ist das Denken in Einflusssphären überhaupt adäquat? Deutet sich darin nicht ein Rechtsimperialismus an, der der Wissenschaft schlecht zu Gesicht steht, oder doch wenigstens eine etwas provinzielle Selbstbezogenheit? Aber natürlich ging es nicht um nationale Selbstvergewisserung im Wettrennen um Verfassungsexporte. Die Rechtsvergleichung beschäftigt sich seit jeher nicht nur mit dem bloßen Vergleich, sondern auch mit den Wechselwirkungen zwischen verschiedenen Rechtsordnungen. Diese Vorgänge der Rezeption und Ausstrahlung prägen schließlich in hohem Maße die geistes- und kulturgeschichtlichen Wurzeln der beteiligten Rechtsordnungen und sind deshalb für ihr Verständnis und Selbstverständnis von großer Bedeutung. Sie bilden übrigens auch die Brücke zwischen Rechtsvergleichung und Rechtsgeschichte. Noch allgemeiner gesprochen werfen sie ein Schlaglicht auf die Pfade und Mechanismen der Entstehung und Weiterentwicklung von Recht. Und konkret gewendet erlauben sie Rückschlüsse auf die Überzeugungs- und Durchsetzungskraft rechtlicher Argumente und Konstruktionen sowie die Bedingungen der Transplantierbarkeit rechtlichen Denkens und rechtlicher Lösungen. Sich Rechenschaft abzulegen, ob die eigenen Produkte exportfähig oder Ladenhüter sind, kann einer selbstbewussten und eher introvertiert veranlagten Rechtswissenschaft wie der deutschen nicht schaden. Das gilt auch für die Verfassungsvergleichung. Ganz bewusst bezog sich die Themenstellung nicht auf das positive Verfassungsrecht, sondern auf das Verfassungsrechtsdenken. Denn auf dieser methodischen Ebene sind die Grundlagen des Staats- und Rechtsverständnisses angelegt, auf die es im Verfassungsrecht so sehr ankommt.

Die Themenstellung diente den Referenten als Ankerpunkt für die Darlegung komplexerer, über den deutschen Hintergrund hinausgehender Rezeptionsvorgänge. Fünf Rechtsordnungen wurden exemplarisch in den Blick genommen. Dreimal ging es um „Fernwirkungen“ über grundlegende rechtskulturelle Grenzen hinweg: Japan (Hiroshi Nishihara: „Die Renaissance des deutschen rechtswissenschaftlichen Denkens im japanischen Verfassungsrecht"), Brasilien (Mônia Clarissa Hennig Leal: „Das brasilianische Verfassungsrecht zwischen US-amerikanischer Institutionalisierung und deutscher Dogmatik“) und Spanien (Antonio López Castillo: „Das deutsche Vorbild in Spanien: Kontinuität oder schwindende Bedeutung? “). Zweimal waren „Nahwirkungen“ zu betrachten, wenngleich solche ganz unterschiedlicher Art: 
Österreich (Konrad Lachmayer: „Eine Sprache, zwei Rechtskulturen: deutsches und österreichisches Verfassungsrechtsdenken") und das Europarecht (Claus Dieter Classen: „Wie deutsch ist das Europarecht?").

Gerade diese letzte Themenstellung, die nicht ohne ein gewisses Vergnügen so pointiert formuliert worden war, bündelt Reiz und Problematik der Frage nach nationalen Einflüssen. So wie gestellt, war sie natürlich nicht beantwortbar. Classen ging die Frage schlaglichtartig an, indem er einige Beispiele herausgriff, bei denen eine deutsche Patenschaft besonders deutlich zu sein scheint: Das Kartellrecht, die Entwicklung des Grundrechtsschutzes, das Verhältnismäßigkeitsprinzip vor allem, daneben auch die optionale Regelung einer subjektiv-rechtlichen Klagebefugnis in der AarhusKonvention. Aber sein Grundtenor war doch, dass es dabei um Impulse, nicht Transplantationen ging, soll heißen: Auch das, was mit deutschen Wurzeln und Etiketten daherkommt, ist im Schmelztiegel des Europarechts wesentlich umgeformt worden. Eine quantifizierbare Aussage zum Einfluss des deutschen Rechts auf das Unionsrechts, so sein Resümee, sei daher prinzipiell nicht möglich. Das ist natürlich keine überraschende Folgerung. Das Europarecht kann seiner Funktion und seinen Entstehungsbedingungen nach kein Abbild des deutschen Rechts und auch kein Baukasten aus nationalrechtlichen Einzelbestandteilen sein - weder auf Ebene des positiven Rechts noch auf derjenigen der methodischen und konzeptionellen Grundlagen, des Rechtsdenkens eben. Deshalb wurde in der Diskussion auch darauf hingewiesen, dass, wer deutsche Vorstellungen festschreiben wolle, das im Prozess der Sekundärrechtsetzung tun müsse und sich nicht darauf verlassen dürfe, der EuGH werde es schon richten. Wenn also diese Grundaussage eindeutig ist, ist dann die Frage nach deutschen Einflüssen überhaupt noch von Belang? Zwei Gründe sprechen dafür: Zum einen geht es im Verhältnis zwischen nationalem und Europarecht ja nicht um horizontale, sondern um vertikale Ausstrahlungswirkungen zwischen Teil- und Gesamtrechtsordnung. Weil das Europarecht unmittelbar in das nationale Recht hineinwirkt, ist umgekehrt die Fähigkeit des nationalen Rechts, das Europarecht mitzuprägen, Teil der integrationspolitischen Gesamtbilanz. Und zweitens spielt die Frage eine Rolle für den Umgang der deutschen Rechtswissenschaft und -praxis mit dem Europarecht. Classen hat auch diesen Punkt angesprochen, die verbreitete Neigung nämlich, das Europarecht „deutsch zu denken“, also in die eingefahrenen Denkmuster der nationalen Dogmatik (und Didaktik) zu pressen. Es ist eines der schwer überwindbaren Defizite der Europarechtswissenschaft, dass ihre Diskurse nach wie vor in relativ hohem Maße national parzelliert sind. Auch hier gilt: Will man dem Europarecht als autonomer Rechtsordnung gerecht werden, muss man sich Rechenschaft darüber ablegen, wie weit das Fundament des gewohnten heimischen Rechtsdenkens trägt. 
Was die horizontalen Ausstrahlungen auf andere nationale Rechtsordnungen angeht, so näherten sich die Referenten diesem Thema ganz unterschiedlich. Für Österreich als dem offensichtlichsten und nächstliegenden Rezeptionskandidaten stand naturgemäß die Frage nach den Unterschieden, nach den Grenzen der Rezeption im Mittelpunkt. Konrad Lachmayer zeichnete hier ein ambivalentes Bild: Einerseits bilden die deutsche Jurisprudenz und Judikatur einen steten Referenzpunkt für die österreichischen Diskurse, sie werden intensiv beobachtet und rezipiert und bilden auch das dominierende Objekt österreichischer Verfassungsvergleichung. Auf der anderen Seite stehen bekannte und grundsätzliche Divergenzen im Verfassungsrechtsdenken, die Lachmayer allerdings weniger auf die rechtstheoretische Fundierung ("Mythos Kelsen") zurückführen wollte als auf die traditionell exekutivorientierte Ausrichtung der österreichen "Verwaltungsverfassung" - und, in jüngerer Zeit, auf deren strukturelle Offenheit für eine direkte und ungefilterte Aufnahme der europäischen Grundrechtssysteme. Einen ganz anderen Zugang wählte Antonio López Castillo für die spanische Perspektive. Er thematisierte nicht den Inhalt, sondern die Pfade der Rezeption und entwarf ein faszinierendes Bild der persönlichen Beziehungen, Netzwerke und Schulen, über die das Gedankengut der deutschen Lehre in die Dogmatisierung des spanischen Verfassungsrechts einfließen konnte.

Intensivere Rezeptionsschübe treten vor allem in politischen Umbruchsituationen oder ihrem Gefolge auf, wenn es notwendig wird, die Staats- oder Rechtsordnung jenseits des rechtskulturell tradierten Bestandes neu auszurichten. In Spanien war der Übergang von der Franco-Diktatur zur Demokratie solch eine Konstellation. Einmal angelegt können derartige Rezeptionspfade sehr langlebig sein, aber auch von parallelen Ausstrahlungen weiterer Rechtsordnungen überlagert werden. Die Referate zu Brasilien und Japan rückten daher beide das Aufeinandertreffen US-amerikanischer und europäisch-deutscher Einflüsse in den Mittelpunkt. Für Brasilien stellte Mônia Clarissa Hennig Leal diese Beobachtungen aus dem Blickwinkel der Verfassungsgerichtsbarkeit an: Ein institutionell nach amerikanischem Muster eingerichtetes Supremo Tribunal Federal, durch verfahrensrechtliche Ergänzungen einer genuinen Verfassungsgerichtsbarkeit angenähert, gepaart mit einem materiellen Verfassungsrecht, das positiv-rechtlich, aber auch durch die vom Gerichtshof eingeschlagenen Dogmatisierungswege wesentliche europäisch-deutsche Anklänge aufweist: Die hervorgehobene Stellung von Menschenwürde und Grundrechten, die Konzeptionen einer objektiven Grundrechtswirkung, grundrechtlicher Schutzpflichten, der Dritt- und Ausstrahlungswirkung, eines Untermaßverbotes und eines rechtlich gewährleisteten Existenzminimums - das waren die genannten Beispiele. Deutlich wurde aber auch, wie vorsichtig solche Rezeptionsvorgänge zu bewerten sind. Das beginnt schon damit, dass ihr Fundament im Einzelfall recht schmal sein kann, wenn sie nur auf wenigen Vermittlerpersönlichkeiten beruht. So wies die Referentin auf einen beachtlichen Rückgriff auf Häberle und seine „offene Gesellschaft der 
Verfassungsinterpreten“ in der Judikatur des Supremo Tribunal Federal hin, aber auch darauf, dass dafür überwiegend eine konkrete Richterpersönlichkeit verantwortlich zeichnet. Auch die Wirkungszusammenhänge sind schwierig zu deuten. Ähnlichkeiten müssen nicht auf Rezeption beruhen, es können auch Parallelerscheinungen sein. Und auch dort, wo eindeutige Referenzen vorliegen, kann ihr Stellenwert unklar sein. Wenn etwa das Numerus-clausus-Urteil des BVerfG in der brasilianischen Rechtsprechung zur Begründung grundrechtlicher Leistungsansprüche herangezogen wird, so ist damit noch nicht gesagt, inwieweit es zu der Weichenstellung tatsächlich beigetragen hat oder nur als ausländische Autorität ein Grundrechtsverständnis unterfüttert, für das der Boden ohnehin bereitet war. Ebenso lässt sich hier wie in anderen Fällen beobachten, dass die Übernahme ausländischer Präzedenzfälle und Doktrinen häufig mit Verzerrungen einhergeht, weil nur einzelne ihrer Kernaussagen herausgegriffen werden - und auch, weil die übernommenen Präzedenzfälle oder Doktrinen in ihrer Herkunftsrechtsordnung möglicherweise nur Randerscheinungen oder schon überholt sind. Auch dafür kann die Numerus-clausus-Entscheidung, die - wie schon in der Diskussion angemerkt wurde - in Deutschland heute so wohl gar nicht mehr ergehen würde, als Beispiel dienen. Rezeptionsvorgänge erfolgen eben typischerweise mit Verzögerung. Dass umgekehrt auch die einmal übernommenen Konzeptionen der Dynamik einer Weiterentwicklung nach den spezifischen Bedürfnissen der aufnehmenden Rechtsordnung unterliegen, versteht sich ohnehin von selbst.

Das gilt mutatis mutandis auch für das Verhältnismäßigkeitsprinzip, das gefühlte Musterbeispiel des deutschen Exportschlagers. Nicht umsonst nahm es in dreien der Referate einen prominenten Platz ein als Exempel für eine deutsche Ausstrahlungswirkung auf das europäische, brasilianische und japanische Recht. Stets war diese Feststellung jedoch von dem caveat begleitet, dass die Identität des Etiketts nicht notwendigerweise eine Identität der Rezeptur bedeutet. Auf die eine oder andere Weise findet man die gewohnten Strukturen und Standards des Verhältnismäßigkeitsprinzips in seinen neuen Umgebungen verändert vor. Dazu trägt auch das Aufeinandertreffen mit der US-amerikanischen Konzeption des gerichtlichen Prüfungsmaßstabs bei. Dieses Zusammentreffen und die daraus resultierende Spannungslage zwischen verschiedenen Rezeptionsquellen hat Hiroshi Nishihara eindrucksvoll für das japanische Recht demonstriert - eine Abfolge phasenverschobener Einflüsse des deutschen spätkonstitutionellen Staatsrechts, des amerikanischen Grundrechtsdenkens und der Judikatur des BVerfG, die zu einem in der japanischen Lehre und Rechtsprechung nach wie vor offenen Schwebezustand zwischen deutscher Verhältnismäßigkeit und amerikanischem two- bzw. three-tier approach geführt hat. Dahinter steht letztlich die Frage, wo das theoretisch angemessene Maß gerichtlicher Kontrolldichte liegt. Ist die gerichtliche Überprüfung staatlichen Handelns nach einem bloßen „Tatsachenfeststellungsmodell“ zu formen, also fokussiert auf die fak- 
tische Erforderlichkeit des Grundrechtseingriffs? Soll sie mit abstrakten Wertungen über die Gewichtigkeit der beteiligten Rechtsgüter operieren, wie es die amerikanische Doktrin tut, oder muss sie zu einer fallbezogenen Güterabwägung schreiten („konkretes Werturteilsmodell“)? Und - diese Frage stellt sich so oder so - welche Einschätzungsspielräume muss die Judikative dabei den von ihr kontrollierten Staatsorganen belassen? Wie immer man dies im Einzelnen beantworten will, die Fragen zeigen, dass hinter einem Bekenntnis zum Verhältnismäßigkeitsprinzip immer noch eine beträchtliche Bandbreite unterschiedlicher Konzeptionen stehen kann.

Einflüsse des deutschen Verfassungsrechtsdenkens sind also - das lässt sich verallgemeinern - nicht einfach quantifizierbar. Vorhanden und rekonstruierbar sind sie gleichwohl, zuweilen auch dort, wo der unbefangene Beobachter sie nicht erwarten würde. Diesen Spuren nachzugehen ist nicht nur im besten Sinne spannend, sondern immer auch ein guter Anlass zur Reflexion über die eigenen Denkmuster. Das lässt sich natürlich auch umdrehen, wie Konrad Lachmayer in seinem Resümee angemerkt hat: Welche Einflüsse anderer Rechtsordnungen finden sich im deutschen Verfassungsrecht, im deutschen Verfassungsrechtsdenken? Auch das wäre - jenseits des offensichtlichen Erbes der französischen und amerikanischen Revolution - einmal eine lohnende Spurensuche. 